\title{
LES COLÉOPTERES PARASITES ACCIDENTELS DE L'HOMME
}

\section{Par Jean THÉODORIDĖS}

L'ordre des Coléoptères, comptant plus de trois cent mille espèces, a fait récemment l'objet d'un excellent ouvrage d'ensemble (Paulian, 1943). A la diversité de formes, s'ajoute aussi celle des milieux habités par ces insectes. L'on peut dire sans exagérer que tout milieu, même défavorable à la vie, possède une faune coléoptérique.

Dans leurs relations avec les autres organismes, les représentants de cet ordre comprennent d'innombrables formes inféodées à des végétaux et comptent, dans leurs rapports avec les autres animaux, des prédateurs, des commensaux (termitophiles et myrmécophiles) et quelques ectoparasites de vertébrés.

L'homme lui-même n'a pas échappé à cette expansion et bien que les cas de pseudo-parasitisme (on ne peut vraiment parler ici de parasitisme, car ce sont des cas accidentels et il n'existe aucun coléoptère strictement parasite de l'homme) soient relativement rares, il existe cependant une assez abondante littérature sur ce sujet qui nous a suggéré cette mise au point.

Si l'on veut essayer de classer les différentes infestations parasitaires (1) provoquées chez l'homme par des coléoptères, l'on aurait par ordre d'importance :

1. Infestation du tube digestif (canthariasis ou scarabiasis).

2. Infestation des organes des sens (œil, voies nasales et auditives).

3. Infestation des voies génito-urinaires.

Nous allons brièvement les passer en revue :

1. - Tube digestif. - Un des coléoptères les plus fréquents dans l'intestin humain est le commun « ver de farine », stade larvaire de Tenebrio molitor L. (Tenebrionida).

(1) Nous ne mentionnerons pas ici d'autres lésions que les Coléoptères peuvent occasionner extérieurement chez l'homme par émission de substances toxiques (dermatites vésiculeuses, conjonctivites, etc...).

ANn, de Parasitologie, T, XXIII, Nos 5-6, -1948 , p. 348-363. 
Hinman et Faust (1932) ont donné un aperçu des principaux cas connus depuis le Xvir siècle (Forestus, 1619) et par la suite : Acrel (1799) trouva 50 larves dans l'estomac d'une femme, Bateman (1811) signala un cas intestinal, Pickells (1824) trouva 50 larves' et 7 adultes, et Riley et Howard (1889) 2 larves ; dans ces deux derniers cas, les «parasites » sont signalés comme étant rejetés d'estomacs de femmes. Brumpt (1927) cite aussi des cas d'évacuation per anum de larves de cette espèce. Hinman et Faust donnent eux-mêmes le compte rendu de deux cas observés : un homme ayant vomi une larve au dernier stade et la découverte d'une autre dans une amygdale.

Plus récemment, Palmer (1946) a signalé le cas d'un nourrisson qui, à partir de l'âge de cinq mois, commença à expulser des larves de Tenebrio par voie rectale. Ceci fut suivi de manque d'appétit, de vomissements et de cris de la part de l'enfant, tandis que des larves étaient toujours passées vivantes malgré l'administration de purges et d'énémas. Des Tenebrio adultes trouvés dans une boìte de céréales confirmèrent le diagnostic, et l'auteur conclut que les larves provenaient d'œufs ingérés accidentellement.

Un autre Ténébrionide: Blaps mortisaga L., a été signalé (in Neveu-Lemaire, 1938) comme ayant été rejeté à l'état larvaire par l'anus, des larves ayant été trouvées vivantes dans les selles.

La deuxième catégorie de coléoptères fréquentant le tube digestif de l'homme comprend certains représentants de la grande famille Scarabaeidæx (d'où le nom de scarabiasis généralement employé pour désigner ces infestations) et en particulier de la sous-famille Coprinæ. Cette infection est surtout répandue dans l'Inde et à Ceylan, où elle a été étudiée par plusieurs auteurs :

Senior-White (1920) signale le cas d'un enfant cinghalais de 4 ans, présentant les symptòmes suivants : légère température, toux, douleurs abdominales, selles liquides, et qui, après l'administration d'un antihelminthique, expulsa dans ses selles des spécimens vivants d'Onthophagus bifasciatus $\mathrm{Hb}$. Cet auteur signale d'autres cas de Ceylan et bien que cette infestation soit peu sérieuse, elle n'en possède pas moins un nom vernaculaire, Kurumini mandama (maladie à coléoptères). Il suppose que les enfants s'infectent en ingérant des œufs ou des larves fréquents dans les poissons pourris dont les indigènes sont friands.

Dans un autre article, le même auteur et Sen (1921) donnent des renseignements complémentaires sur cette infestation, en signalant d'autres cas de scarabiasis dus à $O$. bifasciatus : 
Dey $(1919,1920)$ (1) rapporte des cas survenus dans le Bengale, ainsi que S. C. Sen qui insiste sur le fait que les enfants de 3 à 5 ans se montraient particulièrement sensibles, souffrant de coliques et de diarrhée et présentant un aspect émacié. Chakravarty (1919) signale trois cas dans le Bengale, et Senior-White et Sen supposent que la pénétration des scarabéides ne peut s'effectuer par voie rectale, d'une part le sphincter ne pouvant leur livrer passage, d'autre part du fait que des cas d'infestation se rencontrent chez des adultes propres et bien vêtus, qui auraient remarqué une pénétration per anum. Cés auteurs suggèrent que les coléoptères atteignent peut-être l'entrée du rectum, sans toutefois y pénétrer, lors de la défécation des enfants indigènes, et qu'ils en sont rejetés à la selle suivante. De toute façon, le problème de la respiration des coléoptères adultes et celui de la résistance de leurs œufs aux sucs digestifs (dans l'hypothèse d'une ingestion par voie buccale) restent posés.

Fletcher, lors de la discussion suivant la communication de Senior-White et Sen, fit remarquer le fait que surtout des scarabéides $\$ \uparrow$ avaient été trouvés, ce qui ferait pencher vers une pénétration rectale (peut-être en vue d'y pondre), car autrement les deux sexes devraient être représentés en proportions égales.

Fletcher (1924), dans un aperçu sur la scarabiasis rencontrée dans le Bengale et à Ceylan, insiste aussi sur le fait que les enfants sont plus aptes à contracter cette infection que les adultes.

A O. bifasciatus, signalé par Senior-White (1920), il ajoute une autre espèce indéterminée d'Onthophagus et Caccobius mutans Sharp. Il précise aussi l'écologie normale de ces espèces, toutes coprophages, et cherche aussi à expliquer comment ces insectes peuvent atteindre l'intestin. Il suggère notamment une pénétration directe par voie rectale, pendant le sommeil des indigènes, ceci étant facilité par l'état de propreté douteuse de ceux-ci.

(1) Depuis l'impression de ce travail et grâce à l'obligeance de M. R. L. Sheppard, Secrétaire du \& Bureau of Hygiene and Tropical Diseases » de Londres, j'ai pu obtenir des copies des notes de divers auteurs hindous, ce qui permet d'ajouter les détails suivants :

Dey (1919), dans une lettre très vague, relate l'existence d'un \& ver s'échappant en volant $\gg($ sic) des selles récemment évacuées.

Sen (1919), répondant au précédent, signale qu'il a rencontré des cas analogues dans le Bengale, et q:1'après détermination les insectes rejetés s'avérèrent être des coléoptères coprophages.

Chakravarty (1919) précise les données des aúeurs précédents et signale le cas d'un homme de 40 ans et de ses deux enfants âgés de 6 et 4 ans qui rejetèrent des coléoptères après des injections salines rectales.

Dey (1920) donne des détails sur les cas observés, notamment celui d'un enfant apparemment normal, mais expulsant, le matin, des insectes dans ses selles; l'auteur cite les remèdes utilisés.

Enfin Sen (1924) signale des cas dans le district de Faridpur ainsi qu'à Akyab et demande à ses collègues des précisions sur la manière dont sont contractés les coléoptères. 
Iyengar (1924) signale la présence de C. mutans dans l'intestin. Une fillette de cinq ans, du district de Faridpur (Bengale), rejeta des coléoptères déterminés par Arrow. L'auteur insiste sur l'authenticité incontestable de ce cas constaté de visu.

Le même auteur étudia plus tard (1928) l'infection par d'autres Coprinæ dans le Bengale. C'est peut-être là le compte rendu le plus complet et le plus documenté sur les cas de scarabiasis. L'auteur signale des cas de coléoptères rejetés avec les excréments par des enfants dans le Bengale; ceux-ci furent déterminés comme étant $O$. bifasciatus et $C$. mutans déjà cités.

Les principales localités où des infestations par $O$. bifasciatus ont été observées sont :

Damodya (District de Faridpur, Bengale; Dr S. K. Sil, observateur). Berhamganj (District de Faridpur, Bengale ; $D^{r}$ A. C. Dey, observateur). Faridpur (District de Faridpur, Bengale ; D ${ }^{r}$ A. K. Sarkar, observateur). Akyab (Birmanie, Dr S. C. Sen).

Onthophagus unifasciatus Fabr. aurait été aussi trouvé dans les mêmes circonstances [observations du $\mathrm{D}^{\mathrm{r}} \mathrm{H}$. Prajapati à Hinoo, Ranchi, Bihar, et cas de Matale (Senior-White, 1920) signalé comme étant dû à $O$. bifasciatus ]. Iyengar précise la condition des indigènes atteints d'infestation à $O$. bifasciatus : ce sont généralement des enfants de moins de 10 ans, souffrant de diarrhées et présentant de la flatulence, anorexie et anémie. Les coléoptères rejetés restent une minute ou deux dans les selles, puis s'envolent (de là le nom de "flying worms » ou \& flying intestinal worms » que leur ont donné des médecins de l'Inde, inexactement d'ailleurs, ceux-ci étant des adultes et non des larves). Un des enfants âgé d'un an a été observé expulsant 8 à 10 spécimens par jour.

L'auteur hindou illustre ces données de deux cas précis :

Cas 1. - (Communiqué par le Dr S. C. Sen, Akyab, Birmanie). Un enfant de 4 ans de Chakdaha (Bengale) fut traité pendant quatre mois, mais sans amélioration visible. Les troubles intestinaux étaient caractérisés par une diarrhée à raison de 6 à 7 selles par jour, très fétides, avec mucus. Le malade présentait de plus un aspect pâle et émacié, un appétit vorace et une soif intense. Les selles furent examinées en vue d'y déceler des œufs de schistosomes ou des amibes, mais en vain.

Cas 2. - (Communiqué par le $\mathrm{D}^{\mathrm{r}}$ Prajapati précité). Une fillette de 2 ans, anémiée et présentant un appétit vorace, rejeta des scarabéides par voie rectale devant le praticien. 
Iyengar aborde ensuite le sujet si discuté du mode de pénétration des coléoptères dans l'intestin, reprenant et critiquant les hypothèses formulées antérieurement, qui se réduisent à trois principales :

a. - Des œufs ou larves de coléoptères sont ingérés par voie buccale avec du poisson pourri (Senior-White 1920). Dans ce cas se pose le problème de la résistance de ceux-ci aux sucs digestifs, objection à laquelle Iyengar ajoute une autre pouvant se formuler ainsi :

Si des œufs (ou des larves) étaient ingérés accidentellement, comment se fait-il que l'on ne rencontre essentiellement qu'une seule espèce à l'état adulte chez les gens infestés, alors que tant de Coprinæx pondent dans la viande et le poisson en décomposition ? De plus, il fait remarquer que des enfants d'un an, ne se nourrissant que de laitages, sont atteints de la maladie.

b. - Les scarabéides pénétreraient dans le rectum, pendant la défécation des indigènes malpropres.

c. - Iyengar reprend l'hypothèse d'une pénétration par voie rectale pendant la nuit, insistant sur le fait que la taille relativement faible des coléoptères en question (en particulier $C$. mutans) permettrait leur passage par le sphincter anal.

Il s'appuie sur des observations antérieures d'Arrow (1920), qui décrivit du nord de l'Australie un nouveau genre de Coprinæ: Macropocopris, dont plusieurs espèces vivent dans la fourrure des kangourous, au voisinage de l'anus de ceux-ci ; certains spécimens furent même trouvés dans le rectum des marsupiaux. La biologie de ces Coprides pourrait expliquer la pénétration d'autres membres de ce groupe (Onthophagus, Caccobius), dans l'intestin humain, par voie rectale.

Iyengar, enfin, note le fait que chez certaines espèces de Coprides, les sexes sont difficiles à distinguer, par suite d'un dimorphisme très peu accentué, ce qui expliquerait que des iq soient plus fréquemment incriminées.

En ce qui concerne les cas de scarabiasis en Afrique, Poulton (1921) rapporte à la Société entomologique de Londres que différentes espèces d'Onthophagus et de Sisyphus avaient été envoyées de Natal pour détermination, ayant été soi-disant expulsées vivantes dans les selles d'une femme, à la suite de l'ingestion d'une médecine indigène.

Une indigène du Zoulouland aurait aussi rejeté pendant plus de 10 ans des spécimens vivants d'Aphodius marginicollis. Mais Poulton conclut qu'il ne s'agit là que de mystifications de la part des « médecins » indigènes. Il critique aussi les assertions de Senior- 
White (1920) prétendant que, d'après Arrow, l'éminent spécialiste des Scarabéides coprophages, deux des figures de $O$. bifasciatus représentées n'appartiendraient pas en fait à cette espèce et qu'il s'agirait de deux autres espèces et concluant que les cas de scarabiasis à Ceylan demandent confirmation. Enfin Botha de Meillon (1937) signale qu'un indigène aurait expulsé, à l'hôpital de Eshowe (Natal), avec ses selles, des coléopì̀res vivants appartenant au genre Onthophagus. Cette maladie serait bien connue des indigènes, sous le nom de "Ikambi lentelo ».

Faute de plus grande précision, il semble qu'il faille garder toute réserve sur ces cas de scarabiasis sud-africains.

Il semble cependant qu'il ne faille pas généraliser et que si les " cas » sud-africains semblent fictifs, il n'en est pas moins vrai que les travaux de Senior-White et Iyengar ne laissent aucun doute sur l'authenticité des cas observés dans l'Inde et à Ceylan.

D'autres cas sporadiques d'ingestions accidentelles de coléoptères et de lésions subséquentes ont été signalés :

Sandberg (1890) rapporte le eas d'un enfant de 10 ans souffrant de céphalée, de douleurs de l'estomac, de nausées et de crises intestinales suivies d'un épuisement général. Sous l'action d'un vermifuge, des oxyures furent rejetés par voie rectale, mais sans amélioration, puis, après un laxatif, deux larves au dernier stade $(3 \mathrm{~cm}$. de long) d'Agrypnus murinus (= Adelocera murina L.) (Elateridæ) furent éliminées vivantes. L'une d'elles vécut 14 jours plus tard, conservée dans du fumier, mais sans effectuer de pupaison. D'après la taille de ces larves et la durée du cycle de cette espèce, l'auteur conclut qu'elles devaient être présentes dans l'intestin depuis plusieurs années et qu'elles provenaient d'œufs ingérés par l'enfant se promenant dans la nature et ayant dû sucer ses doigts d'une propreté douteuse.

R. Blanchard (1893) rapporte le cas d'un enfant ayant vomi une larve de coléoptère au Sénégal ; il s'agit d'une larve de $8 \mathrm{~mm}$., déterminée comme appartenant au genre Opilo (Cleridæx), représenté au Sénégal par trois espèces $(O$. cinctus Klug., $O$. dimidiatus Casteln., o. gigas Casteln.), celle-ci vivant normalement sous les écorces et ayant dû être ingérée accidentellement avec des substances végétales avariées.

Sharp (1903) signale un coléoptère inséré dans la paroi intestinale. Un vieillard décédé à l'âge de 73 ans révéla à l'examen postmortem deux masses noirâtres sous la muqueuse de la partie inférieure de l'iléon. Le nodule supérieur, une fois sectionné, se trouva contenir un coléoptère vivant déterminé comme étant Otiorrhynchus

ANN, de Parasitologie, T. XXIII, $\mathrm{x}^{\circ 5} 5-6 .-1948$. 
tenebricosus auct. (non Herbst) (= O. clavipes Bonsd.); celui-ci se trouvait entre la muqueuse et les autres tissus de la paroi intestinale, sans qu'il y ait cependant kyste.

L'auteur anglais insiste sur le fait que le coléoptère se trouvait sous la muqueuse et non dans la lumière ou dans un pli de l'intestin. O. tenebricosus (Curculionida), espèce phytophage dont la larve se nourrit de racines et l'adulte de feuilles, a dû être ingéré accidentellement, comme c'est le cas d'autres insectes (ex. Piophila casei parmi les diptères). En tout cas, à propos de cet article, il est à préciser que le décès n'a été nullement causé par la présence du coléoptère.

Enfin, Poirson (1917) signale un cas de pseudo-parasitisme par larves de charançons : un enfant de 7 ans, atteint de téniasis, en Tunisie, vomit, après absorption d'extrait éthéré de fougère mâle, non seulement le tænia, mais aussi six larves de Curculionides indéterminables.

2. - Organes des sens. $a$. - Eil. - Boulai (1908) et surtout Houlbert (1910) relatent le cas unique de parasitisme accidentel de l'œil humain par un coléoptère : une jeune fille de 14 ans se présenta à la consultation du $\mathrm{D}^{r}$ Boulai, de Rennes, se plaignant de douleurs à l'œil gauche ; celui-ei observa en effet une petite tumeur à la surface externe de la sclérotique et une seconde au-dessous de l'iris, recouverte normalement par la paupière inférieure.

La malade se plaignant de démangeaisons et du fait que la tumeur « remuait dans l'œil », cette tumeur fut ouverte et « une sorte de petite larve rosée » de $8 \mathrm{~mm}$. environ s'en échappa. Celleci fut déterminée comme étant une larve de Necrobia (Cleridæ), probablement $N$. violacea L. commun en Bretagne, bien qu'il puisse s'agir aussi de $N$. ruficollis Fabr. L'infestation, d'après ces auteurs, s'expliquerait de la façon suivante : les larves de Necrobia vivant dans des monceaux d'os frais, la jeune fille, de par ses occupations habituelles, aurait souillé ses doigts et les aurait portés à son œil, y véhiculant ainsi des œufs ou des jeunes larves du coléoptère. Houlbert prétend que si la larve n'avait pas été délogée de la tumeur, elle y aurait sans doute achevé ses transformations ; ce serait là le premier et unique cas signalé d'une larve de coléoptère dans l'œil humain.

b. - Voies nasales. - Des cas d'infestation nasale par des coléoptères ont été souvent signalés, mais il faut se méfier des mystifications fréquentes des indigènes ; par exemple, Drake-Brockman (1913) rapporte le cas d'une vieille femme somali prétendant qu'un 
coléoptère avait pénétré dans son nez lors d'un sommeil dans la jungle et que, quelques mois plus tard, après avoir ingéré un remède préparé par une autre vieille femme, " experte pour débarrasser les gens de leurs coléoptères $»$, elle aurait rejeté 41 petits coléoptères. La potion preserite comprenait du mouton bouilli, auquel était ajoutée la racine d'une certaine plante, et, d'après la « patiente », l'odeur de viande aurait attiré les coléoptères'du nez à l'estomac, d'où ils auraient été vomis : Mais le plus curieux est que (d'après elle), chaque fois qu'elle va à la còte et qu'elle absorbe ce remède, elle rejette des coléoptères toujours vivants.

Voulant vérifier ces assertions plus que douteuses, l'auteur endormit la patiente au chloroforme pour la débarrasser de ses derniers coléoptères, mais, bien qu'elle vomit à plusieurs reprises, aucun de ces derniers ne se montra. La conclusion sur ce « cas » est aussi nette qu'édifiante: les-spécimens soi-disant rejetés s'avérèrent appartenir à deux espèces distinctes !

Par contre, Liggett (1931) signale un cas authentique et beaucoup plus intéressant : une fillette de 15 ans se présenta à la clinique laryngologique de l'Université de New-York, se plaignant de troubles des voies nasales (abondance de mucus et de pus rejetés par le nez) et signalant qu'elle avait' expectoré à plusieurs reprises \& un insecte vivant à nombreuses pattes, brun noir et d'environ $1 \mathrm{~cm}$. de long », précédé de « vers entièrement différents », petits, blanes et apodes. L'examen clinique révéla une sinusite maxillaire bilatérale et les parasites furent supposés se trouver là. L'incision chirurgicale des antrums révéla dans ceux-ci des polypes, du tissu nécrotique et du pus, mais aucun parasite n'y fut découvert. Une ponction suivie d'irrigation des sinus sphénoïdaux ne donna pas de meilleur résultat. Mais à la suite de ces interventions, la patiente en se mouchant émit trois insectes vivants et, en répétant les irrigations deux fois par semaine, chaque fois une ou deux larves furent rejetées. Le traitement fut ensuite effectué par huile nasale.

La larve se révéla être celle d'Attagenus piceus Oliv. (Dermesti$d æ$ ), coléoptère s'attaquant aux tapis, matelas et couvertures ; ces larves sont remarquables en ce qu'elles mettent deux ans avant d'atteindre l'état d'imago.

Les autres cas d'infestation du nez sont attribuables à des myiases causées par des larves de diptères (voir Leclercq, 1948), et c'est là le seul cas connu où un coléoptère en soit la cause.

c. - Voies auditives. - Forsius (1913) relate le cas d'un ouvrier se plaignant de picotements dans l'oreille gauche, avec abondance de cérumen. L'on supposa tout d'abord une scarlatine, mais un 
examen attentif révéla dans l'oreille la présence d'un fragment de coléoptère d'un $1 / 2 \mathrm{~cm}$., qui put être déterminé comme étant Leistotrophus murinus L. (= Ontholestes murinus L.) (Staphylinidx), espèce normalement saprophage. L'insecte avait pénétré accidentellement dans l'oreille dix ans auparavant et le patient avait essayé de le retirer avec une brindille de bois, mais en vain : le coléoptère était resté et ce sont ses débris momifiés qui avaient causé une légère enflure et une abondante sécrétion de cérumen.

Le même auteur signale à ce propos que Sahlberg avait démenti l'assertion suivant laquelle Callidium violaceum L. (Cerambycidx) aurait causé la mort d'un prêtre en pénétrant dans son oreille.

Deux auteurs espagnols : Lopez-Neyra et Roselly (in NeveuLemaire, 1938), ont signalé la présence d'Acinopus picipesøe (Carabidæ) dans l'oreille d'une femme ; il y avait détruit la membrane du tympan et envahi la caisse tympanique.

3. - Voies génito-urinaires. - Tulpius (1652) signalait déjà une larve de coléoptère (probablement Tenebrio molitor), trouvée dans la vessie d'un patient.

Archibald et King (1919) ont publié le cas d'un indigène du Soudan anglo-égyptien qui, souffrant de mictions difficiles et douloureuses, ainsi que d'hématurie, fut hospitalisé et examiné. Des œufs de schistosomes dans l'urine confirmèrent la bilharziose et, malgré les soins prodigués à l'hôpital, les symptômes de strangurie, dus vraisemblablement à une obstruction du conduit génito-urinaire, continuèrent. L'urètre fut donc lavé avec une solution de permanganate de potassium et de nombreuses larves de coléoptères furent rejetées par le méat urinaire. Un autre lavage, le jour suivant, donna le même résultat et ceci fut suivi d'un soulagement immédiat et de la guérison totale du malade.

La larve n'a pas pu être déterminée avec certitude, bien que l'on pense qu'il s'agisse d'un Cleridæ. Les auteurs n'arrivent pas à expliquer comment ces larves ont pu pénétrer dans l'organisme ; il nous semble qu'une ingestion accidentelle d'œufs ou de jeunes larves soit vraisemblable, à moins qu'il ne s'agisse d'un cas de pénétration directe dans les voies génito-urinaires, comme dans le cas suivant:

Sternberg (1926) relate le cas d'un homme qui, atteint d'urétrite à la suite d'une blennorragie, se plaignait de douleurs au moment des mictions. En pressant la partie antérieure de l'urètre, une sérosité plus ou moins claire put être obtenue, où cependant aucun gonocoque ne put être décelé à la suite des essais classiques. Puis, un jour, une petite larve de $5 \mathrm{~mm}$. 5 fut expulsée avec l'urine, suivie trois jours plus tard d'une autre ; lors de l'émission de la première 
larve, le patient ressentit une légère douleur, comme si celle-ci passait à travers un rétrécissement, et l'auteur conclut qu'elle devait se trouver encastrée dans la paroi urétrale et que l'orifice de communication avec l'urètre devait être trop étroit. La larve fut déterminée comme étant celle de Niptus holoceucus Fald. (Ptinidæ), coléoptère commun dans les maisons.

L'auteur explique la présence de ces larves de la manière suivante : des œufs, déposés sur le bord de la canule servant aux lavages, avaient été entrainés dans l'urètre, lors du traitement de la gonorrhée au protargol (albumose argentique titrant 8,3\% d'argent), ceci étant confirmé par le fait qu'un grand nombre d'insectes peuvent résister à des solutions chimiques normalement toxiques (Pulex irritans survit après 24 heures d'immersion dans une solution de sublimé à $1 / 500$ ).

Sternberg fit une expérience complémentaire pour vérifier cette hypothèse : une douzaine de canules de verre lavées avec une solution de protargol furent placées à un endroit donné dans une pièce ; deux jours après, au bord de trois des canules, se trouvaient de petits œufs blanc-jaunâtre d'environ $1 \mathrm{~mm}$. et d'autres plus petits sur deux autres.

L'ouverture des canules était placée de telle manière que les coléoptères pouvaient les atteindre sans difficulté. D'autre part, l'auteur observa, au bord et à l'intérieur d'un bécher rempli d'une solution diluée de protargol et placé à proximité des canules, plusieurs coléoptères vivants, les uns étant $N$. holoceucus et les autres appartenant au genre Ptinus de la même famille.

Des œufs furent introduits dans l'urètre d'un témoin pour confirmer le rôle pathogène du coléoptère, et, d'après l'irritation ressentie par celui-ci, Sternberg conclut que les larves mettaient huit jours à se développer dans l'urètre à partir de l'œuf. Une larve de $N$. holoceucus rejetée avec l'urine du témoin 10 jours après le début de l'expérience confirma entièrement le diagnostic de Sternberg.

Dans un travail antérieur, Scott (1921) signalait le fait que Trigonogenius globulum Solier, voisin de $N$. holoceucus par sa position systématique et sa biologie, vivait dans l'argol (résidu se trouvant dans les tonneaux et contenant jusqu'à $80 \%$ de bitartrate de potassium) et que $N$. hololeucus avait été trouvé à l'état larvaire et imaginal dans du cacao en poudre. Ceci montre l'adaptation remarquable des Ptinidæ à des milieux relativement défavorables à la vie.

Pour être complet, il conviendrait aussi de noter une citation d'Aldovrand (1642) (in Paulian, 1943), mentionnant un scarabée rejeté par l'utérus d'une femme. 


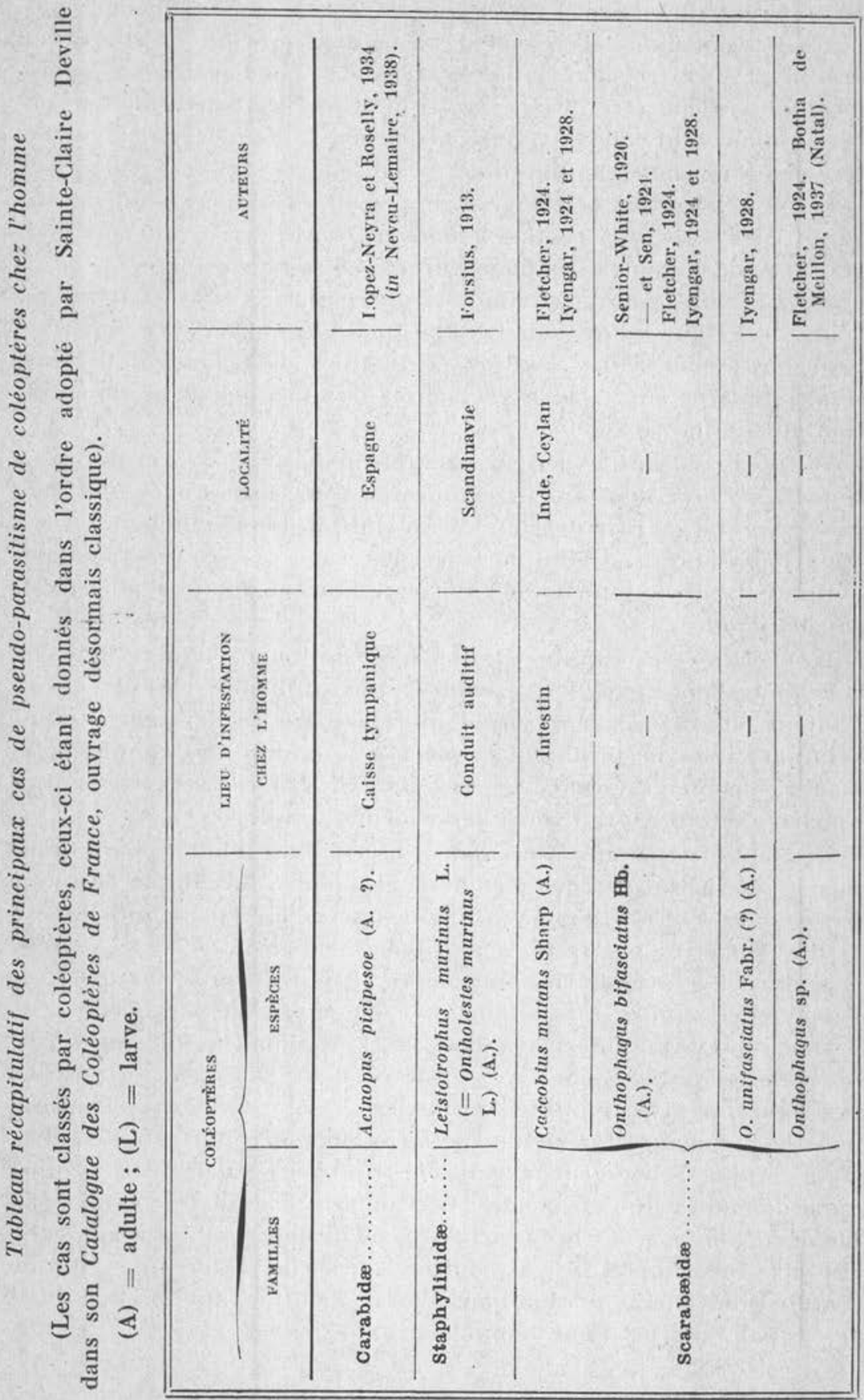




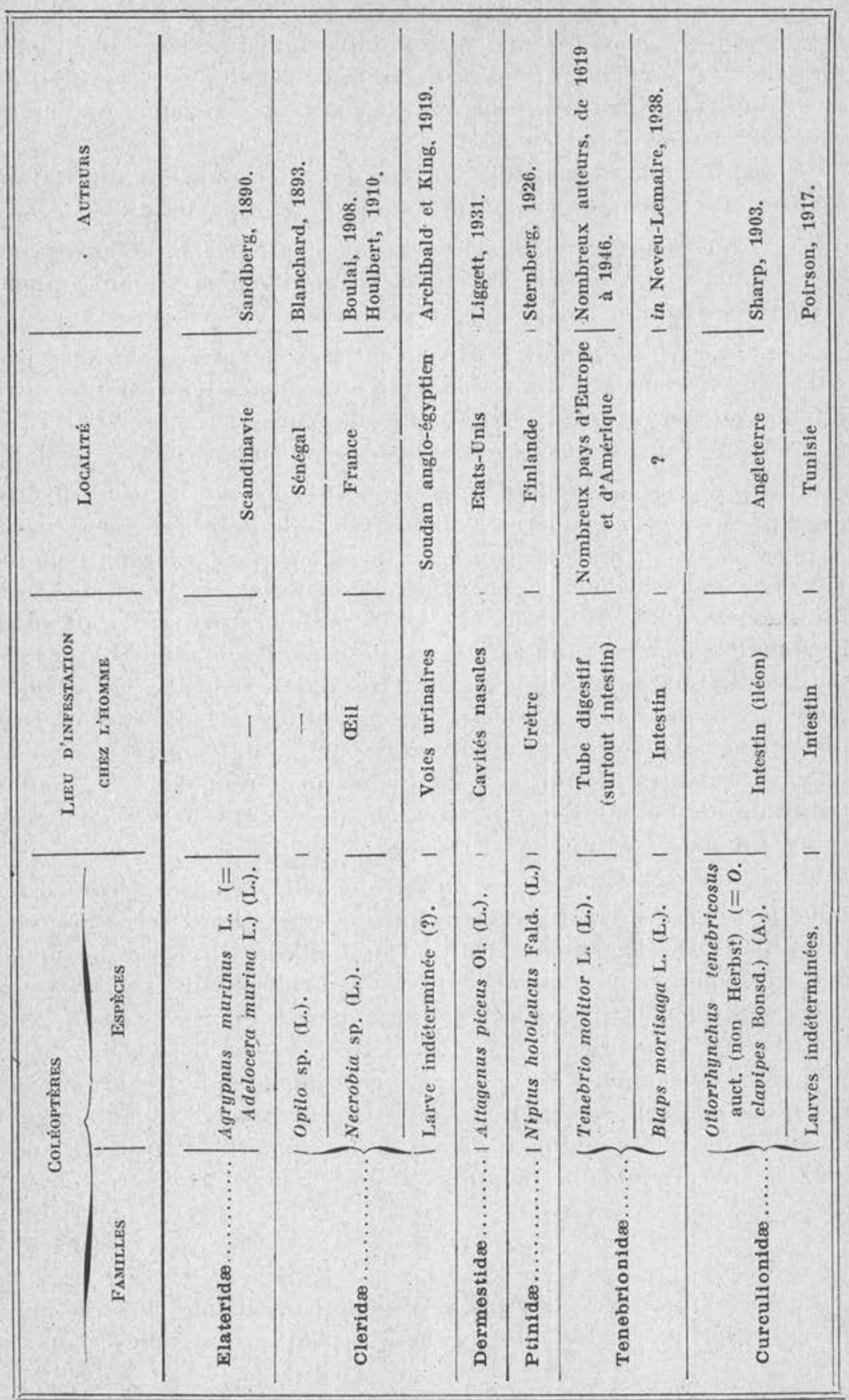


Ainsi l'on voit, par ces différents cas de pseudo-parasitisme, que les coléoptères jouent quand même un certain rôle en parasitologie humaine. Le tableau ci-joint (voir tableau) donne en récapitulation la liste des principales espèces rencontrées, où neuf familles de coléoptères sont représentées.

S'il est difficile de conclure sur des cas sporadiques d'infestation accidentelle, quelques remarques s'imposent cependant :

1. - La fréquence relative des cas de canthariasis à Tenebrio et de scarabiasis à Coprinæ (Caccobius, Onthophagus) donne quand même une certaine importancs médicale à ces coléoptères.

2. - Il est à noter que bien souvent les coléoptères pseudo-parasites ont été rencontrés chez des individus déjà atteints d'une autre infestation parasitaire (schistosomose, oxyurose, téniasis). Ceci est peut-être le fait du hasard, mais mérite cependant d'être constaté.

3. - Les cas de pseudo-parasitisme de coléoptères peuvent être expliqués si l'on considère l'écologie normale de ces derniers :

Nous avons surtout des espèces saprophages (ex. Cleridæ), coprophsges (Scarabaeidx), ou bien des commensaux de l'homme (Dermestidæ, Ptinidæ, Tenebrionidæ). Les premiers peuvent s'attaquer accidentellement à l'homme, du fait de leur régime alimentaire normal, consistant en substances animales ou excréments, les seconds par suite de leur présence dans nos habitations. Quant aux espèces phytophages (Elateridæ, Curculionidæ), elles sont ingérées accidentellement avec des aliments végétaux ou pénètrent dans les cavités naturelles de l'homme (oreille, nez, etc...), à la faveur de séjours de celui-ci dans la nature.

4. - L'on ne saurait non plus trop mettre en garde contre les mystificateurs [surtout des indigènes, comme dans les \& pseudocas » de Drake-Brockman (1913) et de Poulton (1921)] et les malades mythomanes, prétendant rejeter des coléoptères par diverses voies, au mème titre d'ailleurs que des « orvets, serpents, ou lézards » (E. Brumpt).

Nous nous sommes bornés, ici, à ne signaler que les principaux cas que l'on peut considérer comme authentiques, les auteurs les ayant relatés nous semblant dignes de foi, malgré le caractère quelquefois invraisemblable des infestations.

\section{RÉSU MÉ}

Bien que très rares, des cas de pseudo-parasitisme chez l'homme par des coléoptères larvaires ou adultes ont été signalés dans la 
littérature parasitologique et médicale ; ils sont rassemblés et classés ici en trois groupes principaux :

1. - Coléoptères causant des infestations du tube digestif (canthariasis et scarabiasis des auteurs). Il s'agit ici surtout des larves de Tenebrio molitor L., espèce cosmopolite, et de certains scarabéides (Coprinæ) de l'Inde et de Ceylan, dont les adultes se rencontrent dans l'intestin. Dans ce dernier cas, ce sont surtout des enfants qui sont atteints et le mode de pénétration est fort discuté ; il semble cependant que celle-ci s'effectue lors de la défécation des indigènes malpropres.

2. - Certains coléoptères (surtout à l'état larvaire) ont été signalés dans les organes des sens (infestation de l'œil par Necrobia, du nez par Attagenus piceus $\mathrm{Ol}$.).

3. - Enfin, deux cas remarquables d'infestation des voies génitourinaires ont été enregistrés dans la littérature médicale (larve supposée de Cléride dans le Soudan anglo-égyptien et larves de Niptus holoceucus Fald. en Finlande).

\section{ENgLish Summary}

Pseudo-parasitism of larval or adult Coleoptera on man, though very rare, has been now and then recorded and a review of the principal cases is given here. These are classified according to three groups :

1. - Beetles oceurring in the digestive tractus (canthariasis and scarabiasis) ; we are dealing here chiefly with the larvae of Tenebrio molitor L. a cosmopolitan species and some Scarabeids (Coprinæ) from India and Ceylon. These latter enter the digestive tractus in the adult stage and are passed out with the faeces. They occur chiefly in native children and it seems that penetration takes place during defecation.

2. - Some larval Coleoptera have been recorded from the eye (Necrobia) and the nose (Attagenus piceus Ol.).

3. - Finally two remarkable cases of occurrence of beetles in the human uro-genital tractus have been quoted in the medical literature : a supposed larval Clerid in the Anglo-Egyptian Sudan and larvae of the Ptinid Niptus hololeucus Fald. in Finland.

Remerciements. - Qu'il me soit permis d'exprimer ici ma reconnaissance la plus sincère à $\mathrm{M}$. le Prof. E. Brumpt, qui a bien voulu 
accepter cette note pour les Annales de parasitologie humaine et comparée.

Je ne peux manquer non plus d'adresser ici tous mes remerciements au D $\mathrm{D}^{\mathrm{r}}$ J. C. Bequaert (Cambridge, Massachusetts), qui, lors de mon séjour aux Etats-Unis, m'a apporté une aide précieuse en me communiquant ses nombreuses références bibliographiques.

Enfin, il me parait opportun de mentionner ici les conseils que le très regretté $\mathrm{D}^{r} \mathrm{C}$. Desportes m'avait prodigués lors d'un séjour à la station de Richelieu et qui m'ont incité à entreprendre ce travail.

\section{BiBLIOGRAPHIE}

Acres. (J. G.). - Historia vermium larvarum nec non insectorum. Nova Acta Reg. Soc. Sci. Upsal, VI, 1799, p. 98-116.

Aldovrand. - Monstrorum Historia, 1642, p. 219.

AвChibald (R. G.) et King (H. H.). - A note on the occurrence of a coleopterous larva in the urinary tract of a man in the Anglo-Egyptian Sudan. Bull. Entom. Res., IX, 1919, 255-56.

Arkow (G. J.). - A peculiar new genus of Australian beetles. Ann. Magaz. Nat. Hist., (9), VI, 1920, 434-37.

Bateman (T.). - An account of two species of insects discharged from the human body. Edinburgh Med. Surg. Journ., VII, 1811, 41-48.

Blanchard (R.). - Sur une larve de coléoptère vomie par un enfant au Sénégal. Ann. Soc. Entom. France, 1893, Bull., pp. CLXVI-XVII, et Le Naturaliste, X, 2。 série, $1893,107$.

Bотна oe Meillon. - A Note on Two Beetles of Medical Interest in Natal. South Afric. Med. Journ., July 10, 1937, pp. 479-81.

Boula (J.). - Curieux parasite de l'œil. Clin. Ophtalm., XV, 1908, 135.

Brumpt (E.). - Précis de Parasitologie, $4^{\circ}$ édition, Paris, Masson et Cie, 1927.

Сhakravarty (S. K.). - Flying insects from the rectum. Ind. med. Gaz., LIV, $1919,478$.

DEY (A. C.). - Worms. Ind. med. Gaz., LIV, 1919, 318

- Flying worms. Ind. med. Gaz., LV, 1920, 157.

Drake-Brockiman (R. E.). - A \& case $\gg$ of scarabs in the nasal passages. Journ. Lond. Sch. Trop. Med., II, 1913, 173.

Fietcher (T. Bainbrigge). - Intestinal Coleoptera. Ind. med, Gaz., LIX, 1924, 296-97.

Fonest Us (A.). - Opera omnia, Libri I-X, 476 pp., 1619.

Fonsius (R.). - Om twenne fall av insekter i yttre hörselgången hos manniskan. Medd. Soc. Faun. Flor. Fenn., XXXIX, 1913, 139-42.

Hinman (E. H.) et Faust (E. C.). - The ingestion of the larva of Tenebrio molitor L. (mealworm) by man. Journ. of Parasit., XIX, 1932, 119-20.

Houlbert (C.). - Sur une larve de coléoptère (Necrobia Latr.) parasite de l'œil humain. Arch. Parasit., Paris, XIII, 1910, 551-54.

Iyengar (M. O. J.). - Occurrence of a coprid beetle Caccobius mutans Sharp. in the human intestines. Rept, Proc. 5th Entom. Meet. Pusa (1923), 1924, p. 201-202, 1 pl.

Infestations of the human intestines by coprid beetles in Bengal. Ind. med. Gaz., LXIII, 1928, 365-68, 1 pl. 
Langeron (M.) et Rondeau de Noyer (M.). - Coprologie microscopique. Paris, Masson et Cie, 2' édition, 1930.

Leclehce (M.). - Les Myiases. Rev. Méd. Liége, III, 1948, 133-40.

Liggetr (H.). - Parasitic infestation of the nose. Journ. Americ. Med. Assoc., XCVI, 1931, 1571-72.

Neveu-Lemane (M.). - Traite d'Entomologie médicale et vétérinaire. Vigot, Paris, 1938.

PALMer (E. D.). - Intestinai canthariasis due to Tenebrio molitor. Journ. of Parasit., XXXII, 1946, 54-55.

Pauliax (R.). - Les Coléoptères. Payot, Paris, 1943.

Pickells (W.). - Case of a young woman who has discharged from her stomach a number of insects in different stages of their existence. Trans. Ass. King's \& Queen's Coll. Phys. Ireland, 4, 1824, 189-214.

Poirson (H.). - Un cas de pseudo-parasitisme intestinal par larves de charançons. Bull. Soc. Path. Exot., Paris, X, 1917, 385.

Pountos. - Coprid beetles believed to be internal parasites of man. The viles of « medicine-men ». Trans. Entom. Soc..Lond., 1921, LXVI-XVII.

RILEY (C. V.) et HowaRD (L. O.). - Larva of Tenebrio molitor in a woman's stomach. U.S. Dept. Agric. Div. Insect Life, I, 1889, 378-380.

Sandeerg (G.). - Et tilfälde af coleopterlarvers tilhold i tarmkanalen hos et menneske. Entom. Tidskr. Stockholm, XI, 1890, 77-80.

Scotr (H.). - The Ptinid beetle Trigonogenius globulum Solier breeding in argol. Bull. Entom. Res., XII, 1921, 133-34.

SEN (S. C.). - Flying intestinal worms. Indian med. Gaz., LIV, 1919, 396.

- Intestinal Coleoptera. Ind. med. Gaz., LIX, 1924, 112.

Senion-White (R. A.). - On the occurrence of Coleoptera in the human intestine. Ind. Journ. Med. Res., VII, 1920, 568-69, pl. LV.

- et SEN (S. K.). - Further notes on the occurrence of Coleoptera in the human intestine. Rept. Proc. 4th Entom. Meet. Pusa (1921), 1921, 236-39.

SHARP (D.). - A coleopterous insect imbedded in the wall of the human intestine. Proc. Cambridge Philos. Soc., XII, 1903, 199-200.

StennerRg (H.). - Ein Tierparasit in der männlichen Urethra. Klin. Wochenschrift, V, 1926, 229-30.

Tulpius (N.). - Observationes Medica. Lib. II, c. 51 ; Lib. IV, c. 12, 1656.

Biological Laboratories, Harvard University, Cambridge, U.S.A. et Laboratoire d'Entomologie Coloniale du Muséum, Paris. 